\title{
G-Contractive Sequential Composite Mapping Theorem in Banach or Probabilistic Banach Space and Application to Prey-Predator System and A \& H Stock Prices
}

\author{
Tianquan Yun \\ Department of Engineering Mechanics, School of Civil Engineering and Transportation, \\ South China University of Technology, Guangzhou, China \\ E-mail: cttqyun@scut.edu.cn \\ Received March 21, 2011; revised April 7, 2011; accepted April 10, 2011
}

\begin{abstract}
Theorems of iteration g-contractive sequential composite mapping and periodic mapping in Banach or probabilistic Bannach space are proved, which allow some contraction ratios of the sequence of mapping might be larger than or equal to 1, and are more general than the Banach contraction mapping theorem. Application to the proof of existence of solutions of cycling coupled nonlinear differential equations arising from prey-predator system and A \& H stock prices are given.
\end{abstract}

Keywords: G-Contractive Mapping, Periodic Mapping, Probabilistic Banach Space, Prey-Predator System, Differential Equation of Stock Price

\section{Introduction}

Fixed point theorems play an important role for the proof of existence of solution of equations of algebra, differential, and integral, etc. It has been applied to many areas such as mathematical economics, game theory, dynamic optimization, functional analysis, etc. A lot of research work on fixed point theorems of various mappings on different spaces have been done (see [1,2] and their references). Among these works, the Banach contraction mapping theorem is a basic theorem for many research works. However, the Banach contraction mapping theorem needs a serious restriction, that is, all contraction mapping ratios must less than a constant less than 1 . Instead of this restriction, a loosed restriction which allows some contraction mapping ratios to be greater or equal to 1 but the geometric mean of contraction mapping ratios of the sequential mapping (simplifying as "g-contraction mapping") must less than a constant less than 1, is proposed by the author [2]. In this paper, the iterative g-contraction mapping and periodic mapping theorems in Banach or probabilistic Banach space are proved and application to cycling coupled nonlinear differential equations arising from prey-predator system and A \& $\mathrm{H}$ stock prices are given..

The A-stock market in mainland China is a new de- veloping market and is going to connect the rule with international market. The H-stock market in Hong Kong is a district international stock market. Many companies have their shares in both A- and H-markets, e.g., the China Petrol (601857) and Petro China (HK0857), a significant share in A-stock market. Although there are some papers on computational stock price based on certain model [3,4], however, no paper on quantitative analysis of stock prices on different stock markets has been found. This paper establishes a cycling coupled differential equations of stock prices of A \& $\mathrm{H}$ shares, and uses the theorem to prove the existence of solutions and further more find the solution as well as the preypredator problem.

\section{Main Results}

In the following, let us consider a sequential mapping $\left\{T_{i}\right\}, \quad i \in N=\{1,2, \cdots\}, T_{i}: X_{i} \rightarrow X_{i+1}, \forall X_{i} \subset M, M$ is a probability Banach space, i.e., a complete nonempty metric space satisfied probabilistic requirements.

Definition 2.1. A sequential mapping $\left\{T_{i}\right\}$ satisfied (1) is called the sequential composite mapping.

$$
\begin{aligned}
& T_{i}: X_{i} \rightarrow X_{i+1} \text { or } x_{i+1}=T_{i} X_{i}, \\
& \left(x_{i} \in X_{i} \subset M, i \in N\right)
\end{aligned}
$$


By induction, and define

$$
x_{i+1}=T_{i} \circ T_{i-1} \circ \cdots \circ T_{1} x_{1} \equiv T^{i} x_{1},
$$

where

$$
T^{i} \equiv T_{i} \circ T^{i-1} \text { and } T^{1} \equiv T_{1},
$$

The symbol $F \circ G$ represents the composition of mapping $F$ and mapping $G$.

Definition 2.2. $r_{i}$ shown in (4) is called the contraction ratio of $T^{i}$.

$$
\begin{aligned}
& r_{i}=\sup \left[\mathrm{d}\left(T^{i} x, T^{i} y\right) / \mathrm{d}\left(T^{i-1} x, T^{i-1} y\right)\right] \geq 0 \\
& x, y \in X_{i}
\end{aligned}
$$

where $x, y$ are continuous random variables, $\mathrm{d}(x, y)$ is the distance between $x$ and $y$ in $M$, and $T^{0} \equiv 1$.

Obviously, we have

$$
\begin{aligned}
& \mathrm{d}\left(T^{n} x, T^{n} y\right) \leq r_{1} r_{2} \cdots r_{n} \mathrm{~d}(x, y), \\
& (\forall x, \forall y \in M)
\end{aligned}
$$

Definition 2.3. $s_{i}$ shown in (6) is called the iterative contraction ratio of $T^{i}$.

$$
\begin{aligned}
& s_{i}=\operatorname{Sup}\left[\mathrm{d}\left(T^{i+1} x, T^{i} x\right) / \mathrm{d}\left(T^{i} x, T^{i-1} x\right)\right]>0, \\
& \forall x \in M,
\end{aligned}
$$

Obviously, we have

$$
\mathrm{d}\left(T^{n+1} x, T^{n} x\right) \leq s_{1} s_{2} \cdots s_{n} \mathrm{~d}\left(T^{1} x, x\right), \quad(\forall x \in M)
$$

Definition 2.4 A sequential composite mapping $T^{i}$ is called the g-contraction mapping, if for each $i \in N$, there exits a constant $G$, such that the geometric man contraction ratio $G_{i}$ satisfies.

$$
0 \leq G_{i}=\left(r_{1}, r_{2}, \cdots, r_{i}\right)^{1 / i}<G<1, \quad(\forall i \in N)
$$

Definition 2.5. A sequential composite mapping $T^{i}$ is called the iterative g-contraction mapping $U, U$ if for each $i \in N$, there exists a constant $G$, such that the iterative geometric mean contraction ratio $G_{i}$ satisfies.

$$
0 \leq G_{i}=\left(s_{1}, s_{2}, \cdots, s_{i}\right)^{1 / i}<G<1, \quad(\forall i \in N)
$$

Obviously, condition (9) is weaker than condition (8).

Theorem 2.6. Any sequential composite iterative gcontraction mapping of a complete nonempty metric space $M$ into $M$ has a unique fixed point in $M$.

Proof: Suppose that the sequential composite mapping $T^{i}$ satisfies (9).

Choose any point $x$ in $M$, then ,we have

$$
\mathrm{d}\left(T^{n+1} x, T^{n} x\right) \leq G_{n}^{n}\left(T_{1} x, x\right) \quad(n>0)
$$

By the triangle inequality, we have for $m>n$

$$
\begin{aligned}
& \mathrm{d}\left(T^{m} x, T^{n} x\right) \\
& \leq \mathrm{d}\left(T^{m} x, T^{m-1} x\right)+\mathrm{d}\left(T^{m-1} x, T^{m-2} x\right)+\cdots+\mathrm{d}\left(T^{n+1} x, T^{n} x\right) \\
& \leq\left(G_{m-1}^{m-1}+G_{m-2}^{m-2}+\ldots+G_{n}^{n}\right) \mathrm{d}\left(T_{1} x, x\right)
\end{aligned}
$$

By (9), we have

$$
\begin{aligned}
& \mathrm{d}\left(T^{m} x, T^{n} x\right) \leq\left(G^{m-1}+G^{m-2}+\cdots+G^{n}\right) \mathrm{d}\left(T_{1} x, x\right) \\
& \lim \mathrm{d}\left(T^{m} x, T^{n} x\right) \leq\left[G^{n} /(1-G)\right] \mathrm{d}\left(T_{1} x, x\right)=0, \\
& (m, n \rightarrow \infty)
\end{aligned}
$$

Since, $M$ complete, the sequence $\left\{T^{n} x\right\}$ has a limit z in M. i.e., $T^{n} x=z(\forall x \in M, n \rightarrow \infty)$. This fixed point in unique, if there are two fixed points $z$ and $w$, i.e., $T^{n+1} x=z$ and $T^{n} x=w$, then

$$
\mathrm{d}(z, w)=\mathrm{d}\left(T^{n+1} x, T^{n} x\right) \leq G_{n}^{n} \mathrm{~d}\left(T_{1} x, x\right)=0(n \rightarrow \infty),
$$

i.e., $z=w$.

Obviously, this theorem allows part of contraction ratio $s_{i} \geq 1$ if (9) holds. But the Banach contraction mapping theorem needs each contraction ratio $r$ less than 1 , so this theorem is more general. If $i=1$, only $T_{1}=T$, then Theorem 1 reduces to the Banach contraction mapping theorem.

Definition 2.7. A sequential composite mapping $\left\{T^{j}\right\}$ is called the periodic mapping with period $\boldsymbol{k}$, if

$$
T_{j}=T_{j+n k}, \quad(j=1,2, \cdots, k . n \in N),
$$

and is denoted by $P_{j+1}^{n}$. Where the superscript $n$ denotes the times of cycling, the subscript $j \in K=(1,2, \cdots, k)$ indicates the mapping at the corresponding space $X_{j}$, we have

$$
\begin{gathered}
T_{j}: X_{j} \rightarrow X_{j+1}, \text { or } \\
x_{j+1}=T_{j} x_{j}=T_{j} \circ T_{j-1} \circ \cdots \circ T_{j-k+1} X_{j+1-k} \\
=P_{j+1} X_{j+1-k}, \\
\left(x_{j+1}, x_{j+1-k} \in X_{j+1}\right), \\
P_{j+1}=T_{j} \circ T_{j-1} \circ \cdots \circ T_{j-k+1}, \\
T_{j+n k}=P_{j+1}^{n}=P_{j+1} \circ P_{j+1}^{n-1}=P_{j+1} \circ P_{j+1} \circ \cdots \circ P_{j+1}, \\
P_{j}: X_{j} \rightarrow X_{j+k}=X_{j}, \\
x_{k+j}=P_{j} x_{j},\left(x_{j}, x_{k+j} \in X_{j}\right)
\end{gathered}
$$

Theorem 2.8. Any periodic iterative g-contraction mapping of complete nonempty metric space $M$ has a unique set of $k$ related fixed points in $M$. That is $\exists x^{*}{ }_{j} \in X_{j} \subset M$, such that

$$
P_{j} x_{j}^{*}=x_{j}^{*}, T_{j} x_{j}^{*}=x_{j+1}^{*}, x_{k+j}^{*}=x_{j}^{*},
$$


Proof: For a periodic iterative g-contraction mapping, (7) becomes

$$
\begin{aligned}
\mathrm{d}\left(P_{j}^{n} x, P_{j}^{n-1} x\right) & \leq\left(\prod_{j=1}^{k} s_{j}\right)^{n} \mathrm{~d}\left(P_{j} x, x\right) \\
& \leq\left(G_{k}\right)^{n} \mathrm{~d}\left(P_{j} x, x\right), \quad(\forall x \in M)
\end{aligned}
$$

where

$$
G_{k}=\prod_{j=1}^{k} s_{j}<G<1,
$$

The same treatment as Theorem 1 for each $P_{j}^{n}$ instead of $T^{i}$, we can prove that $P_{j}^{n}$ has a unique fixed point $x_{j}^{*}$, i.e., $P_{j}^{n} x_{j}^{*}=x_{j}^{*}$ then

$$
\begin{gathered}
x_{j}^{*}=P_{j}^{n} x_{j}^{*}=P_{j}\left(P_{j}^{n-1} x_{j}^{*}\right)=P_{j} x_{j}^{*},(n \rightarrow \infty) \\
T_{j} x_{j}^{*}=x_{j+1}^{*}=x_{j+k+1}^{*}, \\
x_{j+k}^{*}=x_{j}^{*}, \quad(j \in K)
\end{gathered}
$$

\section{Applications}

\subsection{Application to the Prey-Predator System Problem in Banach Space}

\subsubsection{Considering the Following Cycling Non-Linear Coupled Differential Equations Arising from a} Model of Prey-Predator System [5]

$$
\begin{gathered}
\frac{\mathrm{d} x}{\mathrm{~d} t}=k_{1} a x-k_{2} x y, \\
\frac{\mathrm{d} y}{\mathrm{~d} t}=k_{2} x y-k_{3},
\end{gathered}
$$

where $x \in X$, and $y \in Y$ are continuous variables, and represent the numbers of prey and predator respectively; $X, Y$ are the sets symbolised the prey, and predator respectively; $a, k_{1}, k_{2}, k_{3}$ are constants. Equation (21) shows that the increasing rate of $x$ is proportional to the product of $x$ and the amount of food $a$; and is decreasing with the product of $x$ and $y$. Equation (22) shows that the increasing rate of $y$ is proportional to the product of $x$ and $y$; and is decreasing with $y$ (natural death).

\subsubsection{The Proof of Existence of Solution of (21), (22) by Theorem 2.8}

Equations (21) and (22) are rewritten as (23) and (24) respectively:

$$
y=\frac{1}{k_{2}}\left[k_{1} a-\frac{\mathrm{d} \ln x}{\mathrm{~d} t}\right]=T_{1}(x),
$$

$$
x=\frac{1}{k_{2}}\left[\frac{\mathrm{d} \ln y}{\mathrm{~d} t}+k_{3}\right]=T_{2}(y),
$$

or

$$
\begin{gathered}
y=T_{1} \circ T_{2} y=P_{1} y, \\
x=T_{2} \circ T_{1} x=P_{2} x,
\end{gathered}
$$

Then, Equations (25) and (26) can be solved by iteration method, we have

$$
\begin{aligned}
& y_{i+1}=P_{1} y_{i}=P_{1} \circ P_{1} \circ \cdots \circ P_{1} y_{1}=P_{1}^{i} y_{1}, \\
& y_{i} \in Y, \quad i=1,2, \cdots \\
& x_{i+1}=P_{2} x_{i}=P_{2} \circ P_{2} \circ \cdots \circ P_{2} x_{1}=P_{2}^{i} x_{1}, \\
& x_{i}=T_{2} y_{i} \in X, \quad i=1,2, \cdots
\end{aligned}
$$

$X, Y$ are Banach spaces. $T_{1}, T_{2}$ are continuous mappings. $P_{1}, P_{2}$ are periodic mappings. Since $T_{1}$, $T_{2}$ are continuous functions, $P_{1}, P_{2}$ as well, therefore it must be bounded. We choice the supper norm as the distance on $X$ and $Y$, i.e.,

$$
\begin{aligned}
& \operatorname{Sup}\left|P_{1}^{i} y\right|=R_{1}, \operatorname{Sup}\left|P_{2}^{i} x\right|=R_{2}, \\
& \text { for } \forall x \in X, \forall y \in Y, \text { and } i \in N=\{1,2, \cdots\},
\end{aligned}
$$

Then, (16) becomes

$$
\begin{aligned}
\mathrm{d}\left(P_{1}^{i+1} y, P_{1}^{i} y\right) & =\left\|P_{1}^{i+1} y-P_{1}^{i} y\right\|=\left|P_{1}^{i+1} y-P_{1}^{i} y\right| \\
& \leq G_{2}^{i} \mathrm{~d}\left(P_{1} y, y\right)<G^{i} \mathrm{~d}\left(P_{1} y, y\right),
\end{aligned}
$$

where the constant $G$ is chosen

$$
G=1 / S<1, S=2 R, R=\max \left[R_{1}, R_{2}\right],
$$

According to Theorem 2.8, $y$ in (25) has a fixed point $y^{*}=P_{1} y^{*}$ in $Y$. Similarly, $x$ in (26) has a fixed point $x^{*}=P_{2} x^{*}$ in $X$, and $x^{*}=T_{2} y^{*}, y^{*}=T_{1} x^{*}$.

\subsubsection{An Exact Solution of (21), (22)}

The solution of (21), (22) had been developed by Lotka (1925) and Vollerra (1926) independently (Refs. 1.7 and 1.8 of [5]). However, we can't find these articles for long ages far from now. Notice that (21), (22) may have no exact solution in general, except the food amount $a$ in a special case. Let us find an exact solution by guest.

Suppose that

$$
y=\exp [b+c \sin \omega t]
$$

Substituting $y$ into (24), we get

$$
x=\left[\omega \cos \omega t+k_{3}\right] / k_{2},
$$

Substituting $x$ into (23), if the food amount $a$ is a special function of $t$ shown in the following form 


$$
a=\frac{k_{2}}{k_{1}}\left[\exp (b+c \sin \omega t)+\frac{-c \omega^{2} \sin \omega t}{k_{2}(c \omega \cos \omega t)}\right],
$$

then, (23) is satisfied, and (32), (33) are solutions of (21) and (22). It should be mention that the supposition of food amount $a$ to be a periodic function is reasonable and confirm with the fact than that of the supposition of $a$ to be a constant.

The exact solution of (21), (22) has a referent meaning for understanding the relationship among food, prey and predator quantitatively. The number of predator $y$ mainly depends on the number of prey $x$; and $x$ mainly depends on the food amount $a . a, x$ and $y$ are periodic functions with the same period $\omega$.

\subsection{Application to the Prey-Predator System Problem in Probabilistic Banach Space}

Equation (21) shows that the growing rate of prey number $x$ is proportional to the product of the food amount $a$ and the number of prey $x$, if other elements keep unchanged. The proportion constant is $k_{1}$. Equation (22) shows that the growing rate of predator number $y$ is inverse proportional to the product of $x$ and $y$, if other elements keep unchanged. The inverse proportional constant is $k_{2}$. And constant $k_{3}$ represents the natural death rate of $y$. It is reasonable and reflect the fact that $k_{1}, k_{2}$ and $k_{3}$ are considered as independent random variables than that of constants. Let $k_{1}=u, k_{2}=v$, $k_{3}=w$ be independent continuous random variables $u$, $v, w$, then, $x=x(t, u, v, w), y=y(t, u, v, w)$, are continuous random variables and satisfy the probabilistic properties, i.e.,

$$
\begin{gathered}
f(x) \geq 0, g(y) \geq 0, \\
0 \leq p(0 \leq x)=F(x)=\int_{0}^{x} f(x) \mathrm{d} x \leq 1, \\
0 \leq p(0 \leq y)=G(y)=\int_{0}^{y} g(y) \mathrm{d} y \leq 1, \\
\int_{-\infty}^{\infty} f(x) \mathrm{d} x=\int_{0}^{x_{M}} f(x) \mathrm{d} x=1, \\
\int_{-\infty}^{\infty} g(y) \mathrm{d} y=\int_{0}^{y_{M}} g(y) \mathrm{d} y=1,
\end{gathered}
$$

where $f, g, F, G$ are the density function, distributed function of $x$ and $y$ respectively; $p$ is the probability.

Comparing $x=x(t) \in X$ and $y=y(t) \in Y$ of (23) and (24) in Banach space with

$x=x(t, u, v, w) \in\left[0, x_{M}\right] \subset M$ and

$y=y(t, u, v, w) \in\left[0, y_{M}\right] \subset M \quad$ in probabilistic.

Banach space $M$, we found that there is no differ- ence between the distances defined by Sup norm, i.e.,

$$
\begin{aligned}
& \|x\|=\operatorname{Sup}|x(t, u, v, w)|=\operatorname{Sup}|x(t)|=x_{M}, \\
& \|y\|=\operatorname{Sup}|y(t, u, v, w)|=\operatorname{Sup}|y(t)|=y_{M},
\end{aligned}
$$

Mappings $T_{1}, T_{2}$ and $P_{1}, P_{2}$ of (25) and (26) in Banach space are continuous mappings, so these mappings are still continuous mappings in probabilistic Banach space ((Lemma 4.3 continuous mapping) of [2]) and the proof of existence of fixed points in Section 3.1.2 is still suited for the case of probabilistic Banach space.

\subsection{Application to A \& H Stock Prices}

\subsubsection{Cycling Coupled Differential Equations of Stock Prices of A \& $\mathbf{H}$ Shares}

Let $x=x(t)$ and $y=y(t)$ be the stock prices of China Petrol (601857) and Petrol China (HK0857) respectively. Follows the set up of differential equation of stock price [3,4], we have:

1) Equations of amount of purchasing and selling of $x(t)$

$$
\begin{gathered}
A_{p}(t)=p_{1} x(t)^{-1}+p_{2}[x(t)-a y(t)], \\
A_{s}(t)=s_{1}[x(t)+a y(t)],
\end{gathered}
$$

where $A_{p}(t)$ and $A_{s}(t)$ are the amount of purchasing and selling respectively; $p_{1}, p_{2}, s_{1}, a$ are constants; (36) shows that $A_{p}(t)$ is inversely proportion to $x(t)$ and proportion to the difference of $x(t)-a y(t)$; (37) shows that $A_{s}(t)$ is proportion to $x(t)+a y(t)$.

2) Assumes that the changing rate of stock price is proportion to the difference of demand and supply, we have

$$
\frac{\mathrm{d} x}{\mathrm{~d} t}=g\left[A_{p}(t)-A_{s}(t)\right]
$$

where constant $g$ keeps the same of dimensions in both sides of (40).

3) Substituting (38) and (39) into (40), we have

$$
y=T_{3}(x)=\frac{p_{1}}{a\left(p_{2}-s_{1}\right)} x^{-1}+\frac{1}{a} x+\frac{1}{g} \frac{\mathrm{d} x}{\mathrm{~d} t},
$$

Similarly, we have

$$
x=T_{4}(y)=\frac{p_{1}}{p_{2}+s_{1}} y^{-1}+\frac{p_{2}-s_{1}}{p_{2}+s_{1}} y-\frac{1}{g} \frac{\mathrm{d} y}{\mathrm{~d} t},
$$

In which, the right hand side of (36) is changed to $p_{1} y^{-1}+p_{2}(a y-x)$, and (39) is unchanged.

Equations (41), (42) are cycling coupled non-linear differential equations, and $T_{3}, T_{4}$ are continuous mappings. Similarly to (25) and (26), (41) and (42) have 
fixed points $y^{*}=T_{3} \circ T_{4} y^{*}$ and $x^{*}=T_{4} \circ T_{3} x^{*}$.

If the coefficients in (41) and (42) are viewed as random variables, then, $y$ and $x$ are the functions of random variables, like the case in Section 3.2, $y$ and $x$ also have fixed points in probabilistic Banach space.

\subsubsection{An Exact Solution of Speculating Type Differential Equations of Stock Price for A \& H Stocks}

Usually, (41), (42) may have no exact solution. However, for a simplest case, for example, the second term in (38) is much larger than the first term, i.e., the speculation on the difference of stock prices in A \& H stock market (and suppose that there is no limit for money freely to purchase a stock in A or $\mathrm{H}$ stock market) forms a major part of the purchasing volume. In such case, $p_{1}=0$, and (41), (42) become

$$
\begin{gathered}
y=\frac{1}{a} x+\frac{1}{g} \frac{\mathrm{d} x}{\mathrm{~d} t}, \\
x=\frac{p_{2}-s_{1}}{p_{2}+s_{1}} y-\frac{1}{g} \frac{\mathrm{d} y}{\mathrm{~d} t},
\end{gathered}
$$

Equations (43), (44) are cycling coupled differential equations of stock prices in $\mathrm{A}$ and $\mathrm{H}$ stock markets.

Substituting (44) into (43), we have

$$
\frac{\mathrm{d}^{2} y}{\mathrm{~d} t^{2}}+R \frac{\mathrm{d} y}{\mathrm{~d} t}+S y=0
$$

where $R=(g / a)-k g, S=(a-k) g^{2}, \quad k=\frac{p_{2}-s_{1}}{p_{2}+s_{1}}$.

Equation (45) has exact solution, depended on the root of characteristic function $r^{2}+R r+S=0$.

1) when $R^{2}-4 S>0$,

$$
y=C_{1} \exp \left(r_{1} t\right)+C_{2} \exp \left(r_{2} t\right)
$$

where $C_{1}, C_{2}$ are arbitrary constants, and

$$
\begin{aligned}
& r_{1}=\left(-R+\sqrt{R^{2}-4 S}\right) / 2, \\
& r_{2}=\left(-R-\sqrt{R^{2}-4 S}\right) / 2,
\end{aligned}
$$

2) When $R^{2}-4 S-0$,

$$
y=C \exp (r t), r=-R / 2,
$$

3) When $R^{2}-4 S<0$, the characteristic function has complex roots and there is no real meaning for $y$ has complex value and thus is out of discussion.

Similarly, substituting (43) into (44), we have,

$$
\frac{\mathrm{d}^{2} x}{\mathrm{~d} t^{2}}-R \frac{\mathrm{d} x}{\mathrm{~d} t}+\frac{S}{a} x=0,
$$

4) When $R^{2}-4 S / a>0$,

$$
x=C_{3} \exp \left(r_{3} t\right)+C_{4} \exp \left(r_{4} t\right),
$$

where $C_{3}, C_{4}$ are arbitrary constants, and

$$
\begin{aligned}
& r_{3}=\left[-R+\sqrt{R^{2}-4(S / a)}\right] / 2, \\
& r_{4}=\left[-R-\sqrt{R^{2}-4(S / a)}\right] / 2,
\end{aligned}
$$

5) When $R^{2}-4 S / a=0$,

$$
x=C \exp \left(r_{5} t\right), r_{5}=R / 2,
$$

6) When $R^{2}-4 S / a<0, x$ has no real meaning and is out of discussion.

\section{Discussion on the Solution}

We are interested in "what is $a$ " and "how to find $a$ ". $a=x\left(t^{*}\right) / y\left(t^{*}\right)$ is the ratio of A-share price to H-share price at the equilibrium time $t^{*}$. If $x$-ay $>0$, then the "hot money" (money can freely purchase or sell stocks in A and $\mathrm{H}$ markets) rushes into $\mathrm{H}$-stock market to purchase the cheaper shares; if $x-a y<0$, then the hot money rushes into A-stock market to purchase the cheaper shares. And if $x-a y=0$, then it is the equilibrium state, in which no profits can be made by speculating the difference of stock prices in A-stock or H-stock markets.

$a$ is an important value for decision making of operators. But how to find $a=$ ?

From (41), if $\mathrm{d} x / \mathrm{d} t=0$, then, $a=x\left(t^{*}\right) / y\left(t^{*}\right)$. From (42), if $\mathrm{d} y / \mathrm{d} t=0$, then, $x\left(t_{1}\right)=k y\left(t_{1}\right)$. If both stock prices are in equilibrium state at the same time $t^{*}=t_{1}$, then $a=k$.

The determination of coefficients via market data may be referred to [3], or we can directly use the share prices both in temporary equilibrium states (the so-called "Doji” or the Chinese stock market saying "cross star") of $\mathrm{A}$ and $\mathrm{H}$ stock markets at the same time to find $a$. For example, 2008-04-10, a "Doji” for China Petrol (601857) (opening price 17.11, closing price 17.35 RMB) and a near "Doji” for Petrol China (HK0857) (opening price 10.20 , closing price $9.82 \mathrm{HKD}$ ). Then, $a=x / y$ $=17.35 \times 0.88 / 9.82=1.554$. (where 0.88 is the changing rate for $\mathrm{RMB}$ to $\mathrm{HKD}$ )

The analysis of this example might be useful to decision making of operators and is referred [6] for details.

\section{References}

[1] A. T. Bharucha-Reid, "Fixed Point Theorems in Probabilistic Analysis," Bulletin of the American Mathematical Society, Vol. 83, No. 5, 1976, pp. 641-657. doi:10.1090/S0002-9904-1976-14091-8 
[2] T. Q. Yun, "Fixed Point Theorem of Composition G-Conaction Mapping and Its Applications,” Applied Mathematics and Mechanics, Vol. 22, No. 10, 2001, pp. 1132-1139. doi:10.1023/A:1016337014775

[3] J. S. Yu, T. Q. Yun and Z. M. Guo, "Theory of Computational Securities," In Chinese, Scientific Publication House, Beijing, 2008.

[4] T. Q. Yun and G. L. Lei, "Simplest Differential Equation of Stock Price, Its Solution and Relation to Assumption of Black-Scholes Model,” Applied Mathematics and Me- chanics, Vol. 24, No. 6, 2003, pp. 654-658. doi:10.1007/BF02437866

[5] C. W. Gardiner, "Handbook of Stochastic Methods, for Physics, Chemistry and the Natural Sciences,” SpringerVerlag Press, Berlin, 1983.

[6] T. Q. Yun and T. Yun, "Simple Differential Equations of A \& H Stock Prices and Application to Analysis of Equilibrium State,” Technology and Investment, Vol. 1, No. 2, 2010, pp. 111-114. doi:10.4236/ti.2010.12013 